\title{
LA TRADICIÓN REALISTA EN BARBARA PYM*
}

\author{
M." AIDA DIAZ BILD
}

Universidad de La Laguna

La novela realista nace, como se sabe, de la firme creencia de sus creadores en la existencia de un mundo fenoménico común que puede ser descrito a través de métodos empíricos empleados por la historia. De ahí se deriva el que para estos autores la realidad sea algo susceptible de ser ordenado y estructurado y, además, común al lector y al escritor, de modo que el primero es capaz de reconocer fácilmente el contexto social, cultural, etc. en el que se mueven los personajes. En este sentido, la novela realista aspira a reproducir «a slice of life» y entra dentro del concepto de arte como imitación. Todo ello lleva al empleo de técnicas netamente metonímicas en el intento de ocultar en lo posible que se trata de un artefacto literario: solidez de detalles, desarrollo causal de la trama, énfasis en el carácter único e individual de los personajes y acontecimientos, particularidad de tiempo y lugar y consistencia con la historia. En Working with Structuralism David Lodge lo resume perfectamente:

In contrast, the realistic, antimodernist novel... is essentially metonymic: it tends to imitate as faithfully as discourse can, the actual relations of things to each other in space-time. Characters, their actions and the

* La investigación de este trabajo ha sido financiada por la DGICYT del Ministerio de Educación y Ciencia. Proyecto de Investigación n. ${ }^{\circ}$ PS89-0127. 
background against which they perform these actions, are all knitted together by physical contiguity, temporal sequence and logical cause and effect, and are represented in the text by a selection of synecdochic detail - parts standing for the whole ${ }^{1}$.

Esta cita de Lodge describe eficazmente lo que es la escritura de Barbara Pym, a la vez que la inscribe dentro de la tradición realista de la novela anglosajona, tradición que esta autora ha sabido retomar y explotar con gran acierto y destreza.

En una ocasión la dependienta de una librería tratando de dar una explicación a su afición por los libros de Pym, terminó exclamando: «But when I'm reading I feel as if I'm there $»^{2}$. Y es que Barbara Pym, al igual que los grandes maestros de la tradición liberal humanista, logra que se produzca por unos momentos en el lector «a suspension of disbelief» y crea que lo que se le cuenta ha ocurrido o puede ocurrir en la vida real. La propia autora ha criticado en sus notas la falta de plausibilidad en otros autores: «Read More Women than Men by I. Crompton-Burnett and saw no point in it - unreal people and not much of a story" ${ }^{3}$. Este carácter mimético de la literatura ha sido frecuentemente reiterado por Barbara $\mathrm{Pym}^{4}$ y está estrechamente vinculado a dos aspectos fundamentales de su obra. Por un lado, la necesidad de que se produzca una comunicación entre el lector y la escritora, y aquí, evidentemente, tenemos el elemento de reconocimiento presente en toda obra realista,: «So I try to write what pleases and amuses $m e$ in the hope that a few others will like it too» ${ }^{5}$. Por otro, el carácter lineal y la estructura continua de la novela tradicional, en la que la realidad se concibe como una sucesion de momentos ligeramente diferentes unos de otros, pero al mismo tiempo en relación de continuidad y contigüidad. Este elemento cronologico es particularmente importante, porque inserta a Pym dentro de la tradición de los «storytellers», aspecto éste del que la propia autora hace mención en dos de las citas anteriormente recogidas. Obviamente, en una novela en la que la resolución de una serie de acontecimientos juega un papel

1 DAvID LODGE, Working with Structuralism. Essays and Reviews on Nineteenth-and Twentieth-Century Literature, Routledge \& Kegan Paul, Boston, London \& Henley, 1981, págs. 11-12.

2 Robert J. Graham, «The Narrative Sense of Barbara Pym*, The Life and Work of Barbara Pym, ed. Dale Salwak, Macmillan Press, London, 1987, p. 142.

${ }^{3}$ Barbara Pym, A Very Private Eye. The Diaries, Letters and Notebooks of Barbara Pym, Hazel Holt y Hilary Pym ed., Grafton Books, London, 1989, p. 35.

4 Hazel Holt y Hilary Pym, op. cit., pp. 323, 333.

5 Barbara PyM, «Finding a Voice: a radio talk*, Civil to Strangers and Other Writings, Macmillan London, London, 1987, p. 286. 
destacado, la manipulación que el autor haga del elemento temporal será determinante.

Hemos visto hasta ahora, brevemente, cómo los rasgos que Lodge define como particulares de la obra realista están presentes en las novelas de Barbara Pym. Pero hay un elemento que aún no hemos mencionado y que constituye la esencia del arte de esta autora: «Characters, their actions and the background against which they perform their actions...are represented in the text by a selection of synecdochic detail - parts standing for the whole». Si hay un aspecto de la obra de Barbara Pym que haya sido destacado ${ }^{6}$, e incluso frecuentemente criticado, es su amor por el detalle: «l've always liked detail -in fact my love of triviality has been criticised- so perhaps that was something I developed early" ${ }^{7}$. En sus novelas, que Larkin ha definido acertadamente como miniaturas $^{8}$, el detalle cobra una importancia suprema al trascender su propio significado y revelar aspectos fundamentales del mundo que se describe en la novela y, lo que es más importante, de los personajes que lo pueblan. La relevancia del empleo de la sinécdoque y la metonimia en la novela realista viene dada por el hecho de que son figuras basadas en la contigüidad, por lo que contribuyen a crear esa «illusion of life» a la que todo escritor realista aspira, ocultando así la artificiosidad del vehículo literario. Nuestra intención en este artículo es, precisamente, demostrar, por un lado, el papel fundamental que la sinécdoque y la metonimia juegan en la obra de Barbara Pym y, por otro, cómo aun cuando la escritora recurre a elementos metafóricos, los subordina a la contigüidad del contexto, de manera que sus libros no sólo permitan una lectura fácil, sino, como dijo un editor, sean «a pleasure to read" 9 .

Un análisis exhaustivo de todos los elementos de sinécdoque y metonimia presentes en la obra de Barbara Pym sería imposible en un trabajo de este tipo debido a su amplitud. Por ello, hemos decidido centrarnos en el modo en que la autora emplea distintos objetos para caracterizar a los personajes, excluyendo, por tanto, las reacciones, pensamientos, acciones, etc. de los mismos, que también, indudablemente, contribuyen a su caracterización. Uno de los factores

6 JoHn BAYLEY, «Where, Exactly, is the Pym World?». The Life and Work of Barbara Pym, ed. Dale Salwak, pp. 50-7. RoBert J. GrahaM, «The Narrative Sense of Barbara Pym», The Life and Work of Barbara Pym, ed. Dale Salwak, pp. 142-155. JANICE RosSEN, The World of Barbara Pym, Macmillan Press, London, 1987.

7 Barbara PYM, «Finding a Voice: a radio talk». Civil to Strangers and Other Writings, p. 82.

${ }^{8}$ PhILIP LARKIN, «Reputations Revisited...», Times Literary Supplement, 20 January 1977, p. 66; cit. por Janice Rossen en The World of Barbara Pym, p. 179.

9 Barbara Pym, A Very Private Eye. The Diaries, Letters and Notebooks of Barbara Pym, p. 76. 
que mejor definen a una persona en la vida real son los detalles que pueden encontrarse en su casa o habitación y ello es evidente en las novelas de $\mathrm{Pym}^{10}$. La misma Harriet al ir a la casa de Mr. Donne la primera vez es consciente de ello:

Harriet looked eagerly round, searching for those personal touches that make a room interesting. Other curates had lodged here, but this was the first time Harriet had visited Mr. Donne and there were bound to be differences of detail. The first thing she noticed was a large oar, fastened on the wall over the mantelpiece, with photographs of rowing groups hanging underneath it ${ }^{11}$.

También en Quartet in Autumn hay una referencia a esta realidad:

Letty had often found herself doing this, arranging her clothes in the drawers and wardrobe provided and putting out her personal possessions, the things that might give some clue as to what sort of person she was. (p. 78).

Los despachos de Father Thames y Father Bode, en A Glass of Blessings, son un magnífico ejemplo del modo en que el contenido de una habitación delata a su propietario. Mientras que los «great many objects arranged in glassnes:

10 Las novelas de Barbara Pym que vamos a citar aquí corresponden a las siguientes edicio-

Some Tame Gazelle, Jonathan Cape, London, 1950.

Civil to Strangers and Other Writings, Macmillan London, London, 1987.

Crampton Hodnet, Macmillan, London, 1985.

Excellent Women, Pan Books, London, 1989.

Jane and Prudence, Granada, London, 1983.

Less than Angels, Panther, London, 1985.

A Glass of Blessings, Penguin Books, Harmondsworth, Middlesex, 1986.

No Fond Return of Love, Grafton Books, London. 1988.

An Unsuitable Attachment, Grafton Books, London, 1990.

Quartet in Autumn, Macmillan, London, 1978.

The Sweet Dove Died, Grafton Books, London, 1990.

An Academic Question, Macmillan, London, 1986.

A Few Green Leaves, Grafton Books, London, 1988.

11 Some Tame Gazelle, p. 56. 
fronted cabinets and on the mantelpiece» y la enorme mesa hecha de «some dark rich-looking wood» (p. 107) revelan los intereses artísticos así como la opulencia de Father Thames, la simpleza y el mal gusto de los objetos que «adornan» el despacho de Father Bode («a lampshade decorated with an ugly pattern of orange leaves» (p. 109), entre otros), así como el hecho de que no haya «desk» sino «table», indican su procedencia social y medios económicos.

Otro ejemplo es el de Leonora, en The Sweet Dove Died. Su casa es un reflejo de su obsesión por lo elegante con sus "pretty Victorian furniture and objects» (p. 16) y los detalles que hay encima de la mesa de noche son un indicativo más de cómo Leonora extiende la noción de elegancia a todos los ámbitos de la vida: «...no alarm clock marred the wordly charm of her bedside table... her Guerlain cologne, a bottle of smelling salts, soft aquamarine paper tissues, a phial of brightly coloured pills to relieve stress and strain...» (p. 16). Y la fotografía de sus padres ha sido sustituida por la de sus abuelos, porque éstos dan una imagen más distinguida.

Un último y clarificador ejemplo del modo en que el interior de una casa define a sus dueños lo tenemos en Excellent Women, cuando Mildred es capaz de adivinar cómo son sus nuevas vecinas a través de sus pertenencias:

As I approached it $I$ was able to take note of some of its contents which were lying forlornly in the road. There were some oak chairs and a gate-legged table, an embroidered firescreen and a carved chest, the kind of «good» rather uninteresting things that people of one's own kind might be expected to have. I guessed that the owners were probably a couple of women like Dora and myself, perhaps... (p. 225).

La importancia que los personajes conceden a determinadas cosas es otra señal de su personalidad. Así, Mildred refleja su condición de «excellent woman» cuando le comenta a Helena que en la mudanza se pueden perder objetos tan importantes como una tetera o una sartén (p. 8), y Dulcie en No Fond Return of Love duda que pueda haber algún nexo de unión entre ella y Marjorie, teniendo en cuenta que a ésta le gusta el «china donkey» que había en el rastrillo (p. 248). Por su parte, el talante presuntuoso y egocentrista de Aylwin Forbes queda demostrado cuando se nos indican cuáles son los objetos esenciales que lleva en su maleta: pastillas de levadura, polvos para el estómago, un tónico para el pelo y, por supuesto, las notas de su conferencia ${ }^{12}$.

12 No Fond Return of Love, p. 8. 
El interior del bolso de una persona es en la mayoría de las ocasiones el espejo del alma y ello no podía faltar en las novelas de Barbara Pym. Quizás el caso más ilustrativo lo tengamos en $A$ Glass of Blessings:

I amused myself by observing these students,... A tall bearded young man, whose string bag revealed a loaf of bread (the wrapped, sliced kind), a tin of Nescafé and two books from a public library, filled me with a kind of sadness, as if his whole life had been revealed to me by these telling details. (p. 65).

Otro ejemplo sería el contenido del «string bag» de Mildred, que contiene simplemente una barra de pan y una biografía del Cardenal Newman, ambos elementos propios del bolso de una «excellent woman».

Dentro de los objetos hemos querido hacer una breve alusión a la comida y la bebida, por lo mucho que revelan sobre sus «practicantes». En este sentido es altamente ilustrativa la siguiente consideración de Wilmet en A Glass of Blessings:

...and it occurred to me that one could perhaps classify different groups or circles of people according to drink. I myself seemed to belong to two clearly defined circles - the Martini drinkers and the tea drinkers though I was only just beginning to be initiated into the latter. (pp. 52-3).

La misma Caro, en An Academic Question, cuando ve que el compañero de su hermana va a la cocina y vuelve con un vaso de leche, «made a mental note that he was that kind of person» (p. 124). Mildred, en Excellent Women, refleja claramente el carácter anodino y monótono de su vida cuando Rockingham le dice que es un placer estar sentado al sol en Italia y beber una botella de cualquier cosa y ella le contesta que en su caso tomaría té (p. 32). Ianthe ha sido educada a la antigua, como Mildred, $y$, al igual que ésta, considera que el coñac es sólo para casos de emergencia ${ }^{13}$. Por otro lado, Ianthe revela su procedencia social al no beber nada antes de las comidas, sino que prepara directamente la cena al llegar a casa, al contrario de lo que es propio en la «clase

13 An Unsuitable Attachment, p. 129. 
trabajadora». Penelope es, en An Unsuitable Attachment, la antítesis de Leonora y ello es evidente en la elección que ambas hacen de la bebida: mientras que Ianthe acepta un jerez, «the idea of sticking to sherry sounded so very safe and dull that Penelope naturally chose the Martini» (p. 123). En Quartet in Autumn se afirma cómo el modo en que Letty, Edwin y Norman se reparten las latas de Marcia refleja de forma sutil la personalidad de cada uno de ellos: «Edwin chose spam and stewing steak, Letty prawns and peach halves, Norman sardines, soup, butter beans and the macaroni cheese» (p. 216).

Una alteración en los hábitos culinarios puede reflejar un cambio en un personaje. Así, cuando Tom se va a Africa, Catherine, al contrario de otras veces, toma un desayuno más sustancioso para distinguir esta ocasión de las otras en las que Tom se ha marchado al otro continente; la diferencia está en que antes Tom vivía con ella y ahora no ${ }^{14}$. De igual manera, cuando Ianthe descubre que está enamorada de John, llega a casa y rompe la sagrada norma de no beber nada antes de cenar ${ }^{15}$.

Dentro del estudio de los detalles que definen la personalidad, condición social, etc., hemos querido dejar un apartado especial para la ropa, por el lugar esencial que ésta ocupa en la caracterización de los personajes. Como dice Mildred en Excellent Women: «I had to agree that it was lovely material, but the dress was so completely Lady Farmer that I should have hated to wear it myself and swamp whatever individuality I possess" (p. 20). El mismo Tom, en Less than Angels, cuando pasa delante de la casa de su tía, donde se está celebrando una fiesta, mira su ropa y se pregunta: «Was it just his clothes, then, that were keeping him out of paradise? It must be something more than that..." (p. 161). Evidentemente, hay algo más, se trata del cambio que se ha operado en él y que le ha llevado a rechazar el mundo elitista al que pertenece; pero, indudablemente, sus prendas son la señal más evidente de este giro que ha dado a su vida. Y es que, en cierto modo, la ropa que llevamos es como un uniforme que nos identifica. Anthea lo capta diáfanamente en Crampton Hodnet cuando, observando a los jóvenes de buena familia que vienen a estudiar a Oxford, piensa: «Suède shoes, pin-striped flannels, teddy-bear coats and check capsAnthea knew the uniform so well» (p. 211). Y cuando, en A Glass of Blessings, Mary se casa con Father Ransome, enseguida empieza a vestirse como la mujer de un vicario «in her grey coat and rather too sensible hat» (p. 248).

Pero quizás sea en las oposiciones binarias que encontramos en las novelas donde mejor se ejemplifique la caracterización que de una persona lleva a

14 Less than Angels, p. 188.

15 Un Unsuitable Attachment, p. 136. 
cabo un detalle aparentemente irrelevante como la ropa. Harriet/Belinda, Prudence/Jane, Viola/Dulcie, Penelope/Ianthe, Marcia/Letty, etc., todas ellas tienen modos de ser antagónicos y ello donde más claramente se refleja es en su modo de vestir. Mientras que Harriet prefiere trajes ajustados, lleva puesto un corsé, es más atrevida en su elección de estampados y colores y lleva zapatos de tacón alto, Belinda opta por «shapeless, unfashionable garments» ${ }^{16}$, colores más bien mortecinos y «heavy shoes» con "sensible heels». Por otro lado, Jane no se preocupa en absoluto de su aspecto físico y de hecho con frecuencia la vemos con «the kind of coat one might have used for feeding the chickens in» ${ }^{17}$, mientras que Prudence siempre está elegante, incluso cuando se acuesta para dormir, haciendo que el gorro de noche haga juego con el camisón. En el tercer caso, Viola y Dulcie, la primera vez que ambas se encuentran, Viola es consciente del contraste tan enorme entre ambas:

\footnotetext{
She wore a tweed suit and brogued shoes which looked too heavy for her thin legs.

Already half way to being a dim English spinster, Viola thought, conscious of herself «making a contrast», in her black dress, with her pale, rather haggard face and untidy dark hair ${ }^{18}$.
}

Los «sensible shoes» se convierten a lo largo de toda la novela en el símbolo de la personalidad de Dulcie, porque casi todos los que la conocen la clasifican en base a ellos: Viola duda que pueda casarse (p. 14), Laurel considera que no hay nada elegante o interesante en su tía (p. 44) y Maurice, cuando la vuelve a ver en la galería de arte, es consciente de «irrelevant details like her thin legs and sensible shoes» (p. 131) (la ironía de la autora en el empleo de la palabra «irrelevant» es obvia). Ianthe, al igual que Belinda y Dulcie, lleva zapatos con «sensible heels» y en este sentido es interesante destacar cómo este tipo de calzado parece ser el «uniforme» de las «excellent women». El caso de Ianthe es particularmente curioso, porque la primera vez que se nos presenta a este personaje es a través de Daisy que observa sus zapatos y medias ${ }^{19}$. Al igual que en el caso de Dulcie, cuando Sophia examina detenidamente la ropa de lanthe, incluidos, por supuesto, sus zapatos, llega a la rápida conclusión de

\footnotetext{
16 Some Tame Gazelle, p. 48.

17 Jane and Prudence, p. 54.

18 No Fond Return of Love, pp. 6-7.

19 Un Unsuitable Attachment, p. 25.
} 
que en la búsqueda y captura de Rupert, Ianthe no es una amenaza para Penelope, cuyos «pointed shoes with high heels» se convierten también en un símbolo de este personaje a través de su constante repetición. Penelope es, por supuesto, el polo opuesto a Ianthe y esta última no puede evitar sentirse «a little bewildered at Penelope's appearance and unconventional clothes» (p. 38). Y es que Penelope sigue siempre las últimas tendencias y compra sus vestidos en las tiendas de moda.

El último ejemplo que mencionábamos, el de Marcia y Letty, es quizás uno de los más impresionantes. Ya desde las primeras páginas de Quartet in Autumn se establece la gran diferencia existente entre ambas: a Letty se la describe como «a Home Counties type, still making an effort with her clothes» (p. 8), mientras que a Marcia se la define en términos de su «general look of oddness» (p. 8). La propia Marcia nos confiesa un poco más adelante que realmente no le importa su apariencia, «not like Letty, who was always buying new things and worried if she couldn't get a cardigan in the exact shade to match something» (p. 29). Esta afirmación de Marcia es realmente interesante porque su falta de interés por la ropa es sinónimo de su apatía vital, frente a Letty, cuyo esfuerzo por seguir manteniendo una imagen, revela su empeño en aferrarse a la vida. Por ello es tan importante para Letty seguir preocupándose por su aspecto físico aún después de la jubilación, como símbolo de que la vida continúa y hay que seguir luchando, mientras que Marcia descuida aún más su persona, reflejando así su progresivo deterioro físico y mental. Uno de los ejemplos más ilustrativos lo tenemos cuando los cuatro protagonistas se reúnen tras la jubilación de las dos mujeres:

Letty was the first to arrive, wearing her best tweed suit and carrying a new pair of gloves....

It took the others a moment or two to recover from the apparition standing before them. Marcia was thinner than ever and her light-coloured summer coat hung on her emaciated body. On her feet she wore old furlined sheepskin boots and a pair of much damed stockings, and on her head an unsuitably jaunty straw hat from which her strangely piebald hair straggled in elflocks. (p. 129).

La vestimenta puede servir no sólo para indicar la personalidad de una persona, sino también su país de origen, trabajo o condición social. Un ejemplo del primer caso sería Mr. Tilos, en el que todos en el pueblo reconocen enseguida a un extranjero: «After the black hat there could of course be no 
doubt» ${ }^{20}$. Una muestra del segundo caso lo tenemos en el hermano de Deirdre, Malcolm, que deducimos por su «bowler hat and neat flat brief-case» ${ }^{21}$ que trabaja en la City, como así es. En cuanto a la posición social, tenemos dos ejemplos bien claros. El primero corresponde a Less than Angels. Catherine y Deirdre han quedado para almorzar con la hermana de Tom y Elaine y cuando éstas llegan: «It was perhaps humiliating, Catherine felt, that she and Deirdre should be so easily recognized, hatless, in loose tweed coats and flat shoes» (p. 248). El segundo pertenece a An Unsuitable Attachment. Los zapatos de Ianthe son, como ya dijimos anteriormente, "sensible", pero al mismo tiempo reflejan que está acostumbrada a las cosas de calidad en el hecho de que son de «good leather» (p. 25).

La ropa también puede revelar si es posible la afinidad entre dos personas, aún incluso antes de que intercambien las primeras palabras. Belinda sabe perfectamente en cuanto ve a Olivia, la novia de Mr. Donne, que se entenderán bien: «She felt a glow of warm friendliness towards her, perhaps because of her rather plain, good-humoured face, her sensible felt hat, her not particularly well-cut tweed suit and her low-heeled shoes» 22 .

Asimismo, cuando Mildred conoce a Helena es consciente de la diferencia existente entre ambas:

We were, superficially at any rate, a very unlikely pair to become friendly. She was fair-haired and pretty, gaily dressed in corduroy trousers and a bright jersey, while I, mousy and rather plain anyway, drew attention to these qualities with my shapeless overall and old fawn skirt ${ }^{23}$.

Esta última cita nos lleva a un aspecto que es determinante al hablar de la ropa y es el color. Porque lo que define a las personas no sólo es el tipo de prendas que llevan, sino también la tonalidad de las mismas. Acabamos de ver el ejemplo de Helena y Mildred y más arriba el de Viola y Dulcie. Uno de los principales elementos que separa a Viola de Dulcie es el hecho de que la primera va vestida de negro y éste es un color que a lo largo de las novelas de Barbara Pym es el elegido por sus mujeres más elegantes, como son Leonora o Wilmet. Sus «excellent women», sin embargo, optan preferentemente por tonos

\footnotetext{
20 Civil to Strangers, p. 43.

21 Less than Angels, p. 33.

22 Some Tame Gazelle, p. 236.

23 Excellent Women, pp. 8-9.
} 
apagados, como Dulcie, que va vestida con sus «subdued browns and greys» o Jessie que lleva en una ocasión «a shapeless grey cardigan» y en otra un vestido «in dust-coloured Macclesfield silk with a bluish-grey stripe» ${ }^{24}$, o Belinda, que al ir a la tienda de lanas y ver un «clerical grey» piensa que si no le hace un sueter con ella a Hoccleve puede tejerse uno para sí misma porque ese «dull grey» es, en cierto modo, un reflejo de su existencia.

También los colores azules y verdes son los preferidos por estas «excellent women»; tal es el caso de Mary, a la que Wilmet intenta convencer para que cambie y se compre algo negro, o el de lanthe, que se casa de azul. Para ellas es muy difícil cambiar sus hábitos y así cuando en una ocasión Jessie «comete la osadía» de comprar un vestido en «tender leaf green» que se sale de los cánones establecidos por ella, lo mete en el armario, sabiendo que pasarán meses antes de que se atreva a ponérselo.

Un cambio en los hábitos del vestir de una persona puede significar un giro en sus vidas. Así, cuando en Crampton Hodnet Latimer vuelve de sus vacaciones en Francia y se encuentra con Francis en la carretera, éste inmediatamente se da cuenta de que algo ha sucedido: «He noticed that he was wearing a light grey flannel suit and a collar and tie. Had he given up being a clergyman, Francis wondered?» (p. 195). La realidad es que se ha enamorado. En Civil to Strangers, Adam ha dejado de ser el centro de atención en las reuniones con la llegada de Mr. Tilos y ello se evidencia en que ya no lleva su «velvet coat this evening, and looked just like an ordinary English husband» (p. 99). En An Unsuitable Attachment, Penelope decide por muy poco tiempo dar un giro a su existencia y desempeñar el papel de «unmarried woman». Mark y Sophia son conscientes de que hay algo diferente en ella en cuanto la ven: «Her hair was smoother and neater and she wore an ostentatiously simple dark blue dress" (p. 227).

Dentro de este apartado dedicado a los elementos de sinécdoque y metonimia en las novelas de Barbara Pym, quisiéramos mencionar (un análisis detallado resultaría demasiado largo para nuestros propositos) uno de los ejemplos magistrales. En Less than Angels Professor Mainwaring invita a cuatro aspirantes a una beca de investigación a pasar un fin de semana en su casa y a través de toda una serie de pequeños detalles, como son el equipaje que trae cada uno, lo que beben, sus modales en la mesa, sus ideas sobre la antropología y la religión o el modo en que entre los cuatro se reparten el trabajo de un puzzle, el catedrático es capaz de dibujar un perfil exacto y preciso de cada uno de ellos (capítulos 18 y 19).

24 Crampton Hodnet, p. 156. 
Si bien la sinécdoque y la metonimia son características del texto realista, ello no quiere decir que el autor de este género se limite únicamente a ellas, como explica nuevamente David Lodge. El factor metafórico también está presente aquí, pero, en este caso, el empleo que se hace del mismo se subordina siempre a la contigüidad del contexto, preservándose así la «illusion of life». Mientras que una obra eminentemente metafórica tenderá a establecer analogías que rompan con el contexto, el escritor metonímico intentará adaptarlas al mismo y preferirá el símil a la metáfora. El símil, si bien pertenece al polo metafórico, contribuye a atenuar la alteración de las relaciones de contigüidad que produce la metáfora. Cuando decimos que $\mathrm{A}$ es como $\mathrm{B}$ no confundimos lo que está ahí con lo que es meramente ilustrativo, mientras que en la expresión A en cierto sentido es $\mathrm{B}$ tal confusión puede producirse ${ }^{25}$.

En términos semejantes a Lodge se expresa Le Guern en el capitulo VI de La metáfora y la metonimia ${ }^{26}$. Aunque la metáfora y la similitud introducen una imagen (lo que les distancia de la metonimia), sin embargo, hay diferencias entre ambas. Los términos empleados por la similitud no pierden su sentido propio $\mathrm{y}$, por tanto, «la imagen que introducen no puede considerarse como una imagen asociada; intérviene a nivel de la comunicación logica e intelectualizada» ${ }^{27}$. Ello hace que el receptor no perciba ninguna incompatibilidad semántica, al contrario de lo que ocurre en la metáfora. La imagen que introduce la metáfora, al ser una imagen asociada, desligada del pensamiento lógico, posee una mayor fuerza poética y de persuasión: «Podemos señalar la diferencia de los efectos producidos diciendo que la similitud se dirige a la imaginación por medio del intelecto, mientras que la metáfora afecta a la sensibilidad por medio de la imaginación» ${ }^{28}$.

Como proceso intermedio entre la metáfora y la similitud, Le Guern sitúa el símbolo. Éste, al igual que el símil, se basa en una analogía captada intelectualmente y por ello es también inferior a la metáfora en riqueza poética.

La validez de estas apreciaciones de Lodge y Le Guern se ven claramente ratificadas en las novelas de Pym. Siguiendo el esquema símil-símbolo-metáfora, es significativo cómo, en primer lugar, lo que más abunda en la obra de esta autora británica son el símil y el símbolo, es decir, los elementos metafóricos que en menor medida resquebrajan esa ilusión de realidad que el autor quiere crear; y, en segundo lugar, cuando Barbara Pym utiliza la metáfora lo hace de

25 DAvid LodGe, «Metaphor and Context», The Modes of Moden Writing. Metaphor, Metonymy, and the Typology of Modern Literature, Edward Arnold, London, 1979, pp. 111-124.

26 MiChel Le Guern, La metáfora y la metonimia, Cátedra, Madrid, 1976.

27 Michel Le GUERN, op. cit., p. 64.

28 Michel Le Guern, op. cit., p. 66. 
tal modo que ésta se adapte a la contigüidad del contexto y no suponga una ruptura grave para el lector. Son precisamente estos dos aspectos los que pasamos a analizar a continuación.

El símil desempeña múltiples funciones en las novelas de Barbara Pym y analizarlas todas aquí sería imposible, por lo que trataremos de ilustrar las más significativas. Una de ellas es la de describir la relación amorosa entre un hombre y una mujer. Lo curioso en todos estos casos es cómo la autora, fiel a sus costumbres, huye del falso sentimentalismo y hace que la imagen introducida por el símil se caracterice por su trivialidad y banalidad. En Some Tame Gazelle se dice que el amor de Belinda por Hoccleve es «like a warm, comfortable garment, bedsocks, perhaps, or even woollen combinations» (págs. 157-8). En Crampton Hodnet, el amor es «like a twinge of rheumatism in the winter» (p. 204). En Jane and Prudence, Fabian encaja dentro de la vida de Prudence «like a turbaned blackmoor holding a lamp» (p. 226). En Excellent Women la relación amorosa se describe, desde el punto de vista del hombre, «like having something like a large rabbit thrust into your arms and not knowing what to do with it (p. 135). En A Glass of Blessinas la idea de tener un amante se ha convertido para Rowena y Mildred en algo «remote, even comfortable, now. Like morning coffee with a woman friend in a country town» (p. 146). En An Unsuitable Attachment, Rupert recuerda una línea de una poesía el día de la boda de Ianthe, «something about a garland of red roses on the habit of a nunloving her might have been like that» (p. 252). En The Sweet Dove Died, para James hacer el amor con Phoebe es «like an amusing unreal game» (págs. 57-8).

También se emplea el símil para hacer referencia a otros aspectos del comportamiento de la pareja, como es el hecho de que a una viuda como Mrs. Gray le sea fácil encontrar un nuevo marido, porque, si lo has conseguido una vez, es sencillo repetir, «like mending a fuse ${ }^{29} .0$ el sufrimiento que supone para Deirdre salir con Bernard, el amigo de su hermano, en lugar de con Tom, a quien realmente ama, es «like the dentist's drill pressing on a sensitive tooth ${ }^{30}$.

Por otro lado, el símil es una herramienta perfecta para describir la personalidad o el aspecto de una persona. En Crampton Hodnet, Jessie observa a Latimer mientras conversa con Miss Doggett y el símil que introduce para caracterizarlo es muy sutil: "...sat like a handsome, complacent marmalade cat, telling Miss Doggett all his little fads» (p. 25). En Excellent Women, Sister Blatt, montada en su bicicleta, está espléndida «like a ship in full sail» (p. 54), y cuando un día se encuentra con Mildred y le ve mala cara, le dice: «You look like a wet week at Blackpool» (p. 80). Pero quizás los casos más interesantes

29 Excellent Women, p. 142.

30 Less than Angels, p. 50. 
en el empleo del símil para reflejar el modo de ser de un personaje se dan cuando la autora parte de una misma imagen para describir a distintos sujetos. En Crampton Hodnet, Miss Doggett dice que Latimer es «like a finely tuned instrument», mientras que para Jessie es «like an Aeolian harp»; a su vez, Jess está segura de que para Miss Doggett ella es «a harp with broken strings, an old twanging thing that somebody might play in the street» (págs. 77-8). En No Fond Return of Love, Dulcie recuerda los días en que Maurice le decía que ella era buena, "'like bread'... Following up the analogy, she tried to think what Marjorie was like - some kind of fancy cake or 'pastry' seemed to suggest itself immediately, the kind of thing one might start and not be able to finish» (p. 227). En The Sweet Dove Died, James establece una analogía entre Phoebe y un pequeño castillo de porcelana que está resquebrajado, y entre Leonora y una pieza de Meissen sin fallo alguno; y añade: «It would be an amusing game to liken one's friends and acquaintances to antiques» (p. 43).

Asimismo, con mucha frecuencia Barbara Pym acude a personajes literarios, musicales, del mundo de la pintura o la escultura, para reflejar el aspecto físico, los sentimientos, el carácter, etc. de los personajes. Cuando Mr. Paladin, en Civil to Strangers, logra escapar momentáneamente de los acosos de Miss Gay, whe felt like a character in The Faerie Queene, one of the characters who has successfully withstood the temptations of the Bower of Bliss» (p. 36). En Excellent Women, se compara a Mrs.. Gray por su estilo y cara ovalada y pálida con una heroína de una novela eduardina (p. 78), mientras que, en Less than Angels, la madre de Tom con su «massive greyclad figure with its rather small head stood out like a great Henry Moore sculpture in a London park» (p. 175). Cuando Jane espía a Mr. Mortlake, Mr. Oliver y el carnicero, se siente «like some character in a novel by Mrs. Henry Wood ${ }^{31}$ y Wilmet como la heroína de una novela victoriana cuando entra en el cuarto de estar con la cajita que ella supone que es de Piers, escondida en el bolsillo de su traje ${ }^{32}$. Wilmet también ve a su suegra «rather like a character in Greek tragedy, doing some unnatural things» ${ }^{33}$. En An Unsuitable Attachment, cuando por fin Ianthe, Sophia y Mark llegan al restaurante romano, las luces del taxi iluminan a Rupert, Penelope y Basil «standing in front of a doorway, like characters in an opera» (p. 175). En Quartet in Autumn, cuando Letty se cambia de casa se siente «like a governess in a Victorian novel arriving at a new post» (p. 77).

El mundo de la literatura no sólo es la base para describir a un personaje, sino también una situación. Cuando, en Jane and Prudence, al marido de Jane

31 Jane and Prudence, p. 128.

32 A Glass of Blessings, p. 95.

33 Op. cit., p. 219. 
lo trasladan a una nueva parroquia en el campo y cerca de una «cathedral town», Jane piensa que todo va a ser «like a novel by Hugh Walpole» (p. 11) y al encontrarse a Mr. Oliver, Mr. Mortlake y el carnicero discutiendo en la sacristía: «Really, thought Jane, it was like one of those rather tedious comic scenes in Shakespeare -Dogberry and Verges, perhaps- and therefore beyond her comprehension» (p. 130). En A Glass of Blessings, cuando Wilmet consigue reunir suficiente valor para donar sangre, se encuentra con que le ponen al lado a una señora que no deja de hablar en todo el rato: «It seemed like a "stream of consciousness" novel» (p. 77).

El símil es, en ocasiones, el medio ideal para reflejar las realidades de la vida cotidiana:

A party of people sets out on a journey with all its different components like the jumbled up pieces of a jigsaw puzzle, Sophia thought, waiting for something - some event or just the passing of time-to fit them together as a whole ${ }^{34}$.

Quisiéramos mencionar, por último, con respecto al símil, cómo éste es, frecuentemente, una de las principales fuentes de humor e ironía en las novelas de $\mathrm{Pym}^{35}$, rasgo éste que comparte con otros novelistas como, por ejemplo, David Lodge.

Uno de los aspectos más interesantes al estudiar el empleo que Barbara Pym hace del simbolismo, es constatar cómo la autora utiliza una de las técnicas más apreciadas por los autores realistas de todos los tiempos: la repetición. La repetición de una serie de detalles hace posible que se cree un símbolo, sin que en muchas ocasiones el propio lector sea consciente de ello. Hay múltiples ejemplos en la narrativa de Barbara Pym y a algunos se ha aludido ya cuando hablábamos de los «sensible shoes» de Dulcie y los «pointed shoes with high heels» de Penelope. Otro ejemplo semejante lo tenemos en Some Tame Gazelle, donde Belinda repite continuamente con desdén que los trajes de Agatha son de las mejores casas, simbolizando así su rechazo hacia Agatha por considerar que no es la persona apropiada para Hoccleve (ella sí lo hubiera sido) al no ajustarse a lo que se espera de la mujer de un clérigo. En esta misma

34 Un Unsuitable Attachment, p. 139.

35 Charles Burkhart analiza este aspecto en The Pleasure of Miss Pym, University of Texas Press, Austin, 1987, pp. 77-9. 
novela el énfasis en el modo en que Bishop Grote maneja el indicador en la conferencia que da [«tapped his pointer vigorously on the floor» (p. 177), «tapped his pointer on the floor» (p. 180)] es un símbolo de su carácter inflexible. En Crampton Hodnet, en el primer capítulo, se insiste en el frío que hace en el comedor [«cold», «shivered» (p. 2)] y en el cuarto de Jessie, como símbolo de la falta de calor humano que Jessie encuentra en esa casa. Por el contrario, en An Unsuitable Attachment, el énfasis está en la agradable temperatura que hay en la casa de Ianthe: «It was pleasantly warm in the little hall, Rupert thought, noticing the red glow of a paraffin heater, almost like a sanctuary lamp or the lamp that was said to have burnt clear in Tullia's tomb, for close on fifteen hundred years» (p. 68). En el salón también se está a gusto gracias al «coal fire» y ello hace que John exclame que la casa es «cosy» (p. 68) y que Rupert reflexione sobre lo agradable que se está en esta casa. La propia Ianthe siente tener que ir un día de Cuaresma a misa en lugar de volver a casa «to a comfortable evening by the fire» (p. 96). Todo ello simboliza la tremenda humanidad que se respira en el domicilio de Ianthe.

Otro ejemplo, esta vez de Crampton Hodnet, sería la insistencia en los "drab clothes" de Jessie, que se convierten en un símbolo de lo que es su vida. Un caso similar lo tenemos en Excellent Women, donde se reiteran una serie de sinónimos como «indistinguishable» (p. 49), «nothing outstanding» (p. 53), «unpretentious» (p. 65), «dull» (p. 219) o «uninteresting» (p. 231), que constituyen un símbolo de lo que es para Mildred su propia existencia. En $A$ Glass of Blessings se repite la palabra «useless» aplicada a Wilmet, simbolizando así el tipo de vida que ésta lleva y en The Sweet Dove Died leemos hasta la saciedad los términos «elegant» $\mathrm{y}$ «cool» como atributos de Leonora, de manera que se convierten en un símbolo de la superficialidad y vacuidad de este personaje.

Pero, obviamente, no siempre emplea Barbara Pym la repetición como medio para crear un símbolo. Los pequeños detalles aislados, las acciones de los personajes, etc., pueden, asimismo, convertirse en símbolo. En Some Tame Gazelle, Belinda «was overjoyed» al escuchar que la voz del nuevo cura tiene el timbre esperado porque ello simboliza que todo seguirá igual, como ella así lo desea (p. 252). En Jane and Prudence, Jane observa a su marido cuando éste la mira por encima de las gafas y piensa: «Mild, kindly looks and spectacles...this was what it all came to in the end» (p. 52). Estos dos elementos son el símbolo de lo que ha quedado de aquella pasión del principio y, por lo tanto, de lo que ahora es su matrimonio. En A Few Green Leaves, el pajaro de piedra que hay en casa de los vecinos de Marjorie juega también un papel simbólico. Al principio de su relación con Marjorie, Aylwin lo había cogido y se lo había entregado en señal de su amor y durante todo su matrimonio el pájaro 
habla permanecido en el jardín de al lado. Pero cuando Aylwin decide separarse de su esposa y así se lo comunica, la casa se vende y los nuevos vecinos tiran el pájaro de piedra a la basura: la desaparición de la «escultura» simboliza el final de una relación amorosa. Y en Quartet in Autumn, cuando Marcia se enfurece al descubrir que una de las botellas de leche vacías no corresponde a las suyas, sino que Letty se la dio en cierta ocasión, su ira simboliza su deseo de un total aislamiento del mundo, de que nada ni nadie se inmiscuya en su vida.

Hay un elemento que Barbara Pym emplea asiduamente como símbolo del modo de ser y de los sentimientos de los personajes: las flores y las plantas. En Civil to Strangers, la «aspidistra» de Mrs. Gower ha rejuvenecido y ello es un símbolo de lo que le está pasando a ella a raíz de su amistad con Mr. Gay. A Mildred, en Excellent Women, la definen mejor los «catkins and twigs», mas resistentes y duraderos que las mimosas, generalmente producto de un capricho y de muy corta vida, apenas unas horas. En Jane and Prudence Jane considera que su marido y Mrs. Glaze, la mujer de la limpieza, tienen derecho a ver los laureles desde el estudio y la cocina respectivamente, pero ella no. El simbolismo es claro: los dos primeros cumplen con las tareas que se les ha encomendado y, por lo tanto, han de tener su recompensa, pero ella es un fracaso como ama de casa y mujer de clérigo y no se merece nada. En No Fond Return of Love, Paul Beltane es un joven triste e indefenso y, por ello, las flores adecuadas para él son las rosas «the long, pale, thornless kind» (p. 55). En A Few Green Leaves, el día que Aylwin va a casa de su suegra lleva un ramo de flores que están «stiff and unnatural-looking» (p. 87), simbolizando cómo se encuentra el propio personaje. Por otro lado, a Leonora, en The Sweet Dove Died, las flores le florecen más tarde que a los demás, simbolizando así, una vez más, la elegancia que define su vida.

También la acumulación de detalles contribuye a la creación del simbolismo. En el último capítulo de Crampton Hodnet vemos cómo se repite paso a paso lo ocurrido en el primero: está lloviendo, Jessie observa el «monkeypuzzle», es casi la misma hora (sólo diez minutos de diferencia), se escucha la misma emisora. Ello hace que esta sección final se convierta en símbolo de cómo la misma farsa se reproduce año tras año en Leamington Lodge. En A Few Green Leaves hay otro ejemplo. A lo largo de la novela se nos dice que la rectoría no tiene calefacción central, que las flores de la iglesia huelen mal porque nadie las cambia y la persona que tiene que hacerlo no va a misa, que nunca han tenido incienso, que el cementerio está descuidado. Todo ello simboliza el gran declive de la Iglesia.

El empleo de la metáfora en Barbara Pym se caracteriza fundamentalmente por la contiguiidad con el contexto que se da en las analogías que se estable- 
cen. En Crampton Hodnet, Jessie reflexiona cómo el respeto y la estima son «cold, lifeless things - dry bones picked clean of flesh» (p. 94). La analogía es evidente: se nos está hablando de algo sin vida, frío y la asociación con los huesos es obvia. En la misma novela Miss Dogget intenta convencerse de que no es posible que Lady Beddoes haya tenido amantes cuando estaba casada:

...after all, Lady Beddoes lived in Chester Square, and she visualised the smooth, unbroken line of the houses, all joined together, so that their inhabitants must be like one huge family, united in respectability, morality and the perfection of the upper classes. This was England, Miss Doggett's England, and it would have been a great shock to her if she had detected any crack in its façade. (p. 113).

La metáfora expresada en "any crack in its façade» es fácilmente comprensible, porque previamente se nos ha hablado de la perfección de las casas de esta gente como símbolo de su perfección moral y, por lo tanto, la introducción de la analogía no destruye «the illusion of life».

En The Sweet Dove Died, Leonora y James van a una exposición de gatos y éste observa el comportamiento de uno de ellos en una jaula y lo aplica para describir el de los humanos. La contigüidad con el contexto es evidente y el lector no tiene problemas en captar la metáfora. A ello ayuda también en este caso la presencia de un símil:

They had stopped in front of a cage where a cat-like shape shrouded in a cloth lay fast asleep. How much wiser to contract out altogether, James felt, as this creature had evidently done. Or to sit stolidly in one's earth tray, unmoved by the comments of passers-by. Yet too often, like some of the more exotic breeds, one prowled uneasily round one's cage uttering loud plaintive cries. (p. 96).

Este elemento de contigüidad es incluso ratificado por uno de los personajes de Barbara Pym, Emma, en A Few Green Leaves. En una conversación con Christabel en la que ésta menciona la exposición de flores, Emma se fija en que Christabel tiene «buenos huesos» y piensa que tuvo que haber sido guapa: «the worm in the bud, though that wasn't the kind of thought one could put into 
words at a sherry party. No doubt the mention of flowers had suggested the bud and the worm in it..." (p. 57).

A lo largo de estas páginas hemos analizado cómo la predilección que Barbara Pym revela por la metonimia y la sinécdoque y la subordinación que hace de los elementos metafóricos a la contigüidad del contexto la sitúan claramente dentro de la tradición realista. Evidentemente, nuestra intención ha sido la de ilustrar con algunos ejemplos la base teórica de este artículo, pues un estudio exhaustivo de todos los recursos mencionados aquí sería obviamente imposible. Lo que esperamos haber conseguido con este trabajo es demostrar cómo tras esta aparente fachada de simplicidad que encontramos en las novelas de Barbara Pym, está la labor creativa y el arte de una mujer que sabe perfectamente cómo manejar su medio, el lenguaje, para escribir el tipo de novela que ella quiere escribir y así lograr el efecto deseado en el lector. 